\title{
Reliability of The King's Health Questionnaire and the International Consultation on Incontinence Modular Questionnaire (ICIQ-SF) Short Form in assessing urinary incontinence effects in Polish women
}

\author{
Pawel Kieres ${ }^{1}\left(\mathbb{D}\right.$, Katarzyna Skorupska ${ }^{2}$, Jakub Mlodawski ${ }^{3},{ }^{4}$, Marcin Misiek ${ }^{5}$, \\ Wojciech Rokita ${ }^{3}$, Tomasz Rechberger ${ }^{2}$ (D) \\ ${ }^{1}$ Department of Urology, Saint Raphael's Hospital in Czerwona Gora, Checiny, Poland \\ ${ }^{2} 2^{\text {nd }}$ Department of Gynecology, Medical University of Lublin, Poland \\ ${ }^{3}$ Department and Clinic of Obstetrics and Gynaecology, Collegium Medicum Jan Kochanowski University in Kielce, Poland \\ ${ }^{4}$ Department of Obstetrics and Gynecology, Voivodeship Combined Hospital in Kielce, Poland \\ ${ }^{5}$ Department of Gynecologic Oncology, Holycross Cancer Center, Kielce, Poland
}

\begin{abstract}
Objectives: The King's Health Questionnaire (KHQ) and the International Consultation on Incontinence Modular Questionnaire (ICIQ-SF) Short Form are widely used in clinical practice. The aim of this study was to assess the reliability of KHQ and ICIQ-SF in Polish women.

Material and methods: One hundred fifty-five women with urinary incontinence (UI) aged between 19-82 years underwent urodynamic investigation and completed both KHQ and ICIQ-SF. We performed Principal Component Analysis (PCA) using VARIMAX rotation for all questionnaire pieces to estimate the factor structure and construct the validity of the KHQ and ICIQ. PCA results were also confirmed by Spearman's correlations between KHQ and ICIQ items. Moreover, by Cronbach's alpha coefficient (a) we assessed the internal consistency of the KHQ and ICIQ. STATISTICA version 13.1 software (StatSoft, Poland), and open-source R software (version 3.4.4) were used for statistical analysis.

Results: Of the study group, 77 (49.6\%) patients had stress urinary incontinence (SUI), 9 (5.8\%) patients had Urgency, $10(6.45 \%)$ had OAB and 21 (13.5 \%) had MUI. The factor analysis of the KHQ questions showed four main components, and ICIQ-SF- two main components. Correlations between KHQ and ICIQ-SF were from weak (0.1-0.3) to high (0.5-0.7). The KHQ's Cronbach's alpha was 0.93 and the ICIQ- 0.7. The results obtained from the questionnaire forms did not differ among study groups.

Conclusions: The Polish versions of the KHQ and ICIQ-SF questionnaires have good psychometric values and are useful diagnostic tools in the population of urinary incontinent women.

Key words: The King's Health Questionnaire; the International Consultation on Incontinence Modular Questionnaire; urinary incontinence
\end{abstract}

Ginekologia Polska 2021; 92, 12: 850-855

\section{INTRODUCTION}

Over the past decade, many different questionnaires for assessing symptoms in women with UI have been developed and implemented for use. The ideal questionnaire should help to differentiate the type of urinary incontinence (SUI, Urgency, MUI) and reduce the need for invasive and expensive additional tests.
An ideal questionnaire should: 1. have high sensitivity in detecting urinary incontinence; 2 . can differentiate the causes of urinary incontinence; 3 . have adequate accuracy, reliability and infallibility; 4. measure "Health Related Quality of Life (HRQOL) [1]".

Questionnaires based on HRQOL enable a combined assessment of the impact of UI on the physical activity of 
the patients and their mental health and social functioning. They are significant elements of postoperative assessment of patients with UI. Such questionnaires may be generic or condition-specific [1]. The latter are more sensitive than general questionnaires regarding clinically relevant changes and for assessing treatment effects. The clinician must be sure that the number of points acquired in the questionnaire well shows the actual intensity of the patient's symptoms.

Psychometry is dealing with the practical and theoretical aspects of questionnaires that examine the subjective feelings of patients. The psychometric approach in questionnaire construction is based on the study of reliability, validity and normalization of questionnaires, setting standards for test parameters and ensuring objectivity [2].

Individuals who declared UI symptoms fulfilled the King's Health Questionnaire (KHQ). This questionnaire estimates the effect of lower urinary tract symptoms (LUTS) on patients QoL. The structure of the KHQ questionnaire contains 21 questions. Items are divided into two parts: one - General Health and two - Incontinence impact. There are seven sub-domains addressing: RE (Role Emotional), RP (Role Physical), SL (Social Limitations), PR (Personal Relationships), E (Emotions), S/E (Sleep/Energy), and SS (Severity of Symptoms). Large number of points obtained as a result of the questionnaire means a worse QoL for the patient [3].

The International Consultation on Incontinence Modular Questionnaire (ICIQ-SF) Short Form is a questionnaire that estimates the frequency, severity and the impact of UI on QoL in research and clinical practice. It was first introduced in 1999. ICIQ-SF's level of validation according to ICI grades is A - meaning that it is a highly recommended questionnaire. Its validity, reliability and responsiveness to change have been confirmed [1]. The questionnaire consists of four items: Frequency of UI, Amount of leakage, Overall impact of UI and Self-diagnostics. ICIQ-SF questions use 5-point Likert scales to assess the presence or absence of symptoms and their severity. The maximum score that can be obtain from the questionnaire is 21 . The more points patient gets, the worse symptom severity. The Polish version of the questionnaire was obtained from the iciq.net [4].

\section{Objectives}

We aimed to establish the psychometric values of the polish versions of KHQ and ICIQ-SF questionnaires in the Polish population of women suffering from Ul.

\section{MATERIAL AND METHODS}

\section{Translation}

The Polish versions of the KHQ and ICIQ were obtained from the questionnaires' websites $[5,6]$. In the pilot study, we chose 10 women with UI symptoms (confirmed by uro- dynamics) for the tests of the Polish versions of the questionnaires. We did not observe any major issues. Minor problems that appeared were corrected immediately.

\section{Study population and study design}

The study group was selected from patients who were treated in the Outpatient Clinic between June 2017 and December 2018. All participants signed the informed consent form. We collected socio-demographic data such as age, parity, BMI and menopausal status from the patients. Pelvic Organ Prolapse Quantification (POPQ) scale was assessed for all patients [7]. All patients spoke Polish as their native language. We performed the urodynamic examination using Medtronic's Duet Logic G2 [5]. For the interpretation of the urodynamic results we used the definitions and units which were established by the International Continence Society (ISC). Results of the tests performed in the study group, during the visit showed that 77 (49.6\%) patients suffered from SUI, 9 (5.8\%) patients had Urgency, 10 (6.45\%) had OAB and 21 (13.5\%) had MUI. All patients completed both KHQ and ICIQ questionnaires. Local bioethics committee approved the study (KE 0254/41/2016).

\section{Face/content validity}

Feedback from a group of ten women complaining of UI symptoms, and from three doctors- experts in the Urogynecology field was taken. This group reviewed the KHQ and ICIQ outcomes.

\section{Construct validity}

To establish the factor structure and to determine the validity of the KHQ and ICIQ, Principal Component Analysis (PCA) using VARIMAX rotation was performed for all questionnaire questions. We analyzed the information from the entire sample group. PCA results were affirmed by Spearman's correlations between KHQ and ICIQ items.

The internal consistencies of the KHQ and ICIQ questionnaires were evaluated using Cronbach's alpha coefficient (a). Herein, a value greater than 0.7 indicates high reliability.

\section{Statistical analysis}

Statistical analysis was performed using STATISTICA version 13.1 software (StatSoft, Poland), as well as open-source $R$ software (version 3.4.4). $P$ values less than 0.05 were considered significant. The Shapiro-Wilk test was used to assess normality in individual groups. Accordingly, the distribution of variables on the individual axis of the scale did not meet the assumption of a distribution close to normal. The values of medians and quartile ranges were then used to describe the central tendency in the groups. Kruskall-Wallis analysis of variance with Dunn's post hoc test was applied to 
Table 1. Demographic characteristics of the study group patients

\begin{tabular}{|c|c|c|c|c|c|c|c|c|c|}
\hline \multirow{2}{*}{$\begin{array}{l}\text { Type of Ul according } \\
\text { to Urodynamics }\end{array}$} & \multirow{2}{*}{$\mathbf{N}$} & \multirow{2}{*}{$\begin{array}{l}\text { Age } \\
\text { Mean } \pm \text { SD }\end{array}$} & \multicolumn{2}{|c|}{ Menopause } & \multicolumn{5}{|l|}{ POPQ } \\
\hline & & & Before & After & 0 & 1 & 2 & 3 & 4 \\
\hline No UI & 38 & $52.34 \pm 12.31$ & $18(47.4 \%)$ & $20(52.6 \%)$ & $9(23.68 \%)$ & $12(31.58 \%)$ & $11(28.95 \%)$ & 4 (10.53\%) & $2(5.26 \%)$ \\
\hline SUI & 77 & $56.21 \pm 10.41$ & 20 (25.9\%) & $57(74.1 \%)$ & 15 (19.48\%) & $26(33.77 \%)$ & $22(28.57 \%)$ & $12(15.58 \%)$ & $2(2.6 \%)$ \\
\hline MUI & 21 & $56.76 \pm 8.95$ & $3(14.3 \%)$ & $18(85.7 \%)$ & $6(28.57 \%)$ & $5(23.81 \%)$ & $8(38.1 \%)$ & $2(9.52 \%)$ & $0(0 \%)$ \\
\hline Urgency & 9 & $57.33 \pm 6.52$ & $2(22.2 \%)$ & 7 (77.8\%) & $2(22.2 \%)$ & $5(55.56 \%)$ & 1 (11.11\%) & $0(0 \%)$ & 1 (11.11\%) \\
\hline$O A B$ & 10 & $52.8 \pm 16.59$ & $5(50 \%)$ & $5(50 \%)$ & $4(40 \%)$ & $0(0 \%)$ & $5(50 \%)$ & $1(10 \%)$ & $0(0 \%)$ \\
\hline All & 155 & $55.18 \pm 11.05$ & 48 & 107 & 36 & 48 & 47 & 19 & 5 \\
\hline
\end{tabular}

$\mathrm{UI}$ - urinary incontinence; $\mathrm{SUI}$ — stress urinary incontinence; $\mathrm{MUI}$ - mix urinary incontinence; $\mathrm{OAB}$ — overactive bladder; $\mathrm{SD}$ — standard deviation; $\mathrm{POPQ}$ — pelvic organ prolapse quantification

compare the groups. For ICIQ-SF, significance of differences of means between analyzed groups was calculated using one-way ANOVA and the Tukey post hoc test. We employed Kaiser-Mayer-Olkin (KMO) test and Barlett's test to measure sampling adequacy for principal component analysis.

\section{RESULTS}

One hundred fifty-five women with Ul aged between 19-82 years met the inclusion criteria. One hundred seven patients from the study groups were post-menopausal (69\%) and 48 patients (31\%) were pre-menopausal (Tab. 1).

$\mathrm{KMO}$ measure of sampling correspondence was 0.9 for $\mathrm{KHQ}$ and 0.68 for the ICIQ-SF questionnaire, which indicated that PCA is proper for the datasets. Bartlett's test of sphericity $(p<0.005)$ performed for both analyzed questionnaires showed that the interconnection between specific items in each questionnaire was big enough for PCA.

\section{KHQ}

The Polish KHQ version was proved to have good face validity-no major troubles arose during the application and most respondents found it 'comprehensive' and a 'good' measure.

The factor analysis of the KHQ questions showed four main components, with the cut-off criterion at the level of the matrix's own value > 1 (Kayser's criterion), the total main components explained $66 \%$ of the total variance, the factor loadings of individual questions are presented in Table 2. Based on factor analysis, the dimensions of the questionnaire could not be clearly determined. The common dimension of questions $7 \mathrm{a}$ and $7 \mathrm{~b}$ (questions about sleep and fatigue during the day) and $5 \mathrm{a}$ and $5 \mathrm{~b}$ (questions about relationships with partners and sex life) drew attention, other questions formed sets of points in space, hence leaving room for interpretation.

Internal consistency for the questionnaire expressed by Cronbach's alpha was 0.93 - indicating very good reliability.

The scores obtained from the questionnaires' domains for individual groups of urodynamic diagnoses were analyzed. Due to the small number of patients in groups Urgency and OAB- these groups were analyzed together (Tab. 3).
Table 2. Results of Principal Component Analysis (PCA) - factor loadings after VARIMAX rotation for The King's Health Questionnaire

\begin{tabular}{|c|c|c|c|c|}
\hline \multirow{2}{*}{ Questions } & \multicolumn{4}{|c|}{ Factors } \\
\hline & F1 & F2 & F3 & F4 \\
\hline KHQ 1 & -0.513 & 0.157 & 0.019 & 0.376 \\
\hline KHQ 2 & -0.699 & 0.066 & 0.133 & -0.193 \\
\hline KHQ 3a & -0.748 & 0.199 & 0.092 & -0.370 \\
\hline KHQ 3b & -0.754 & 0.219 & 0.058 & -0.349 \\
\hline KHQ 4a & -0.717 & 0.016 & 0.085 & -0.313 \\
\hline KHQ 4b & -0.690 & 0.220 & -0.238 & -0.123 \\
\hline $\mathrm{KHQ} 4 \mathrm{c}$ & -0.771 & -0.512 & -0.114 & -0.301 \\
\hline KHQ 4d & -0.787 & -0.094 & -0.139 & -0.233 \\
\hline KHQ 5a & -0.375 & -0.833 & -0.026 & -0.080 \\
\hline KHQ 5b & -0.296 & -0.866 & 0.003 & -0.057 \\
\hline KHQ 5c & -0.727 & -0.327 & -0.169 & 0.038 \\
\hline KHQ 6а & -0.834 & -0.083 & 0.006 & 0.205 \\
\hline KHQ 6b & -0.811 & -0.052 & -0.009 & 0.291 \\
\hline KHQ 6c & -0.765 & -0.107 & -0.037 & 0.328 \\
\hline KHQ 7a & -0.573 & 0.200 & -0.594 & 0.226 \\
\hline KHQ 7b & -0.638 & 0.188 & -0.577 & 0.123 \\
\hline KHQ 8a & -0.640 & 0.262 & 0.354 & -0.091 \\
\hline KHQ 8b & -0.482 & 0.150 & 0.003 & -0.052 \\
\hline KHQ 8c & -0.537 & 0.131 & 0.435 & 0.261 \\
\hline KHQ 8d & -0.690 & 0.003 & 0.429 & 0.214 \\
\hline KHQ 8e & -0.766 & -0.050 & 0.260 & 0.205 \\
\hline
\end{tabular}

F1-F4 - extracted principal components. Factor loadings values $>0.7$ are marked; KHQ — The King's Health Questionnaire

The only statistically significant difference was the difference on the Sleep / Energy axis between the SUI and $O A B$ groups (patients with $O A B$ achieved higher scores — indicating worse QoL).

\section{ICIQ-SF}

Factor analysis of ICIQ-SF showed that each of the three single-answer questions is a separate dimension of the 


\begin{tabular}{l|l|l|l|l|}
$\begin{array}{l}\text { Table 3. Mean Ranges of the King's Health Questionnaire's domains } \\
\text { in study groups }\end{array}$ \\
\hline \multirow{2}{*}{ Domains } & Mean Ranges \\
\cline { 2 - 5 } & SUI & MUI & OAB & No UI \\
\cline { 2 - 5 } & $\mathbf{n = 7 7}$ & $\mathbf{n = 2 1}$ & $\mathbf{n = 1 9}$ & $\mathbf{n = 3 8}$ \\
\hline GH & 79.01 & 78.76 & 75.31 & 74.86 \\
\hline II & 76.40 & 84.71 & 84.05 & 74.48 \\
\hline RE & 77.83 & 79.38 & 84.84 & 74.14 \\
\hline RP & 79.55 & 69.02 & 88.00 & 74.81 \\
\hline SL & 75.53 & 86.16 & 87.50 & 73.73 \\
\hline PR & 57.38 & 57.75 & 61.32 & 57.78 \\
\hline E & 74.87 & 86.83 & 90.21 & 73.35 \\
\hline S/E & $71.37^{*}$ & 73.33 & $108.10^{*}$ & 78.96 \\
\hline SS & 76.45 & 83.76 & 86.57 & 73.65 \\
\hline U & &
\end{tabular}

$\mathrm{UI}$ - urinary incontinence; $\mathrm{SUI}$ — stress urinary incontinence; $\mathrm{MUI}$ - mix urinary incontinence; $\mathrm{OAB}$ - overactive bladder + urgency; $\mathrm{GH}$ - genera health; II —-Incontinence impact; RE — role emotional; RP — role physical; SL — social limitations; PR - personal relationships; E - emotions; S/E

— sleep/energy; SS — severity of symptoms

Table 4. Results of Principal Componence Analysis (PCA) - factor loadings after VARIMAX rotation for the International Consultation on Incontinence Modular Questionnaire Short Form

\begin{tabular}{|l|l|l|}
\hline \multirow{2}{*}{ Questions } & Factors & F2 \\
\cline { 2 - 3 } & F1 & 0.157 \\
\hline ICIQ-SF 1 & -0.873 & -0.600 \\
\hline ICIQ-SF 2 & -0.790 & 0.401 \\
\hline ICIQ-SF 3 & -0.840 & \\
\hline
\end{tabular}

F1-F2 - extracted principal components. Factor loadings values $>0.7$ are marked; ICIQ — International Consultation on Incontinence Modular Questionnaire

questionnaire. The two identified main components explain $87.9 \%$ of the total variance (Tab. 4).

Internal consistency for the questionnaire expressed by Cronbach's alpha was 0.7 - indicating good reliability.

We performed comparison of the sum of points obtained in the ICIQ-SF questionnaire between urodynamic diagnosis groups. Due to the lack of normal distribution of the variable in the compared groups, the analysis was carried out with the Kruskall-Wallis nonparamentric test (Tab. 5). The difference between the groups turned out to be on the border of statistical significance $p=0.049$. Post-hoc tests showed no differences in multiple comparisons due to their conservative nature (Tab. 6).

We confirmed the results of PCA between particular items by Spearman's correlation coefficient. The analysis indicated good construct validity (Tab. 7). The number of scores obtained in individual ICIQ-SF questions in most cases statistically significantly positively correlates with the score obtained for individual domains of the KHQ question-
Table 5. Results of Kruskala-Wallis for the International Consultation on Incontinence Modular Questionnaire (ICIQ) Short Form, $p=0.049$

\begin{tabular}{|l|l|l|l|}
\hline Type of UI & N & Sum of rang & Mean rang \\
\hline No UI & 38 & 2421.00 & 63.71 \\
\hline SUI & 77 & 6081.50 & 78.98 \\
\hline MUI & 21 & 1977.50 & 94.16 \\
\hline Urgency & 9 & 914.00 & 101.55 \\
\hline OAB & 10 & 696.00 & 69.60 \\
\hline
\end{tabular}

$\mathrm{UI}$ - urinary incontinence; $\mathrm{SUI}$ — stress urinary incontinence; $\mathrm{MUI}$ - mix urinary incontinence; $\mathrm{OAB}$ - overactive bladder

Table 6. Post-hoc test results for the International Consultation on Incontinence Modular Questionnaire Short Form

\begin{tabular}{|l|l|l|l|l|l|}
\hline Type of UI & $\begin{array}{l}\text { No UI } \\
\text { R: 63.71 }\end{array}$ & $\begin{array}{l}\text { SUI } \\
\text { R: 78.98 }\end{array}$ & $\begin{array}{l}\text { MUI } \\
\text { R:94.17 }\end{array}$ & $\begin{array}{l}\text { Urgency } \\
\text { R: 101.56 }\end{array}$ & $\begin{array}{l}\text { OAB } \\
\text { R: } 69.60\end{array}$ \\
\hline No UI & & 0.86 & 0.12 & 0.23 & 1.0 \\
\hline SUI & 0.86 & & 1.0 & 1.0 & 1.0 \\
\hline MUI & 0.12 & 1.0 & & 1.0 & 1.0 \\
\hline Urgency & 0.23 & 1.0 & 1.0 & & 1.0 \\
\hline OAB & 1.0 & 1.0 & 1.0 & 1.0 & \\
\hline
\end{tabular}

$\mathrm{UI}$ - urinary incontinence; SUI — stress urinary incontinence; $\mathrm{MUI}$ - mix urinary incontinence; $\mathrm{OAB}$ - overactive bladder

Table 7. Spearman's correlations between the King's Health Questionnaire's and The International Consultation on Incontinence Modular Questionnaire Short Form items. For all values, $\mathrm{p}<0.05$

\begin{tabular}{|l|l|l|l|}
\hline KHQ items & ICIQ-SF 1 & ICIQ-SF 2 & ICIQ-SF 3 \\
\hline GH & 0,242134 & 0,168815 & 0,386674 \\
\hline II & 0,533956 & 0,324778 & 0,650014 \\
\hline RL & 0,567921 & 0,361053 & 0,634133 \\
\hline RP & 0,473881 & 0,284848 & 0,619828 \\
\hline SL & 0,416549 & 0,330215 & 0,557505 \\
\hline PR & 0,212849 & 0,248416 & 0,329405 \\
\hline E & 0,486781 & 0,342103 & 0,535871 \\
\hline S/E & 0,329795 & 0,174603 & 0,433668 \\
\hline SS & 0,601508 & 0,432133 & 0,570466 \\
\hline
\end{tabular}

$\mathrm{GH}$ - general health; II — incontinence impact; RP — role physical; $\mathrm{SL}$ - social limitations; PR — personal relationships; E - emotions; S/E — sleep/energy; SS — severity of symptoms; KHQ — The King's Health Questionnaire; ICIQ - International Consultation on Incontinence Modular Questionnaire

naire. These correlations range from weak $(0.1-0.3)$ to high (0.5-0.7) according to J. Guilford [8]. The highest positive correlation was found between physical limitation and ICIQ-SF 3, and the severity of symptoms and ICIQ-SF 1.

\section{DISCUSSION}

Each health measuring device must have the features of a correct conceptual and measuring model. These contain 
reliability, validity, responsiveness, interpretability, low reply and administrative charge, have comparable optional forms, and holding proper cultural and language adaptation [6]. Results of the study showed that both analyzed questionnaires (KHQ and ICIQ) are of good reliability. Both KHQ and ICIQ are commonly used in the diagnosis of UI. Studies have shown that coexisting $O A B$ symptoms are common in women who have been diagnosed with urodynamic stress incontinence (USI) [9], hence, it is necessary to enhance clinical urodynamic diagnosis by way of questionnaires [10].

The PCA mathematically reflects the questionnaire's language structure. The original structure which consisted of seven factors, has not been replicated for the Polish KHQ version. Factor analysis of the Polish KHQ questions showed four main components, and these explained $66 \%$ of the total variance. However, the Portuguese version of the KHQ enabled the development of three factors, which unraveled $68 \%$ of the total variance [11]. Those findings can presumably be elucidated by the assumptions presented by Donovan et al., [12]. They imply that cultural factors may influence the interpretation of symptoms and aspects of QoL in different populations. Our results showed high reliability and internal consistency both in individual parts and collectively. We did not observe statistically significant differences between $\mathrm{KHQ}$ answers in each study groups. This outcome indicates that this questionnaire cannot distinguish between patients with different types of UI. The only difference was observed between patients with $O A B$ and SUI in the sleep/energy domain - indicating lower quality of life in patients with $O A B$.

The ICIQ-SF scoring is a reliable method for pre- and post-treatment evaluation of patients with UI. Studies show that significant correlation exists between ICIQ-SF score and urodynamic parameters [13]. Psychometric properties of the questionnaire, including content, construct and convergent validity, reliability and sensitivity to change have been confirmed by several studies [14-16] and agree with our results. We did not observe differences between study groups in the number of points obtain from the ICIQ-SF questionnaire.

Correlation between $\mathrm{KHQ}$ and ICIQ-SF has been previously examined. Malik et al., in women with self-reported MUI, confirmed good correlation between KHQ and ICIQ score $(0.58, p=0.008)$ [17]. Gotoh et al. [18], proved moderate to high correlation between the ICIQ-SF and the KHQ subscales. We observed correlations from weak (0.1-0.3), to high (0.5-0.7) according to J. Guilford [8]. The highest positive correlation was found between physical limitation and ICIQ-SF 3, and the severity of symptoms and ICIQ-SF 1.

\section{CONCLUSIONS}

The Polish versions of the KHQ and ICIQ-SF questionnaires have good psychometric values and are useful di- agnostic tools in the population of urinary incontinent women.

\section{Acknowledgements}

None.

Funding

None.

\section{Conflict of interests}

None.

\section{Ethics approval}

Bioethical committee approval (KE 0254/41/2016).

\section{Consent to participate}

All patients gave written consent to participate in the study.

\section{Consent for publication}

All authors gave consent to publish In Biomed Research International

\section{Availability of data and material}

Data is available with authors.

\section{Code availability}

Not applicable.

\section{Authors' contributions}

P. Kieres: project development, urodynamics, data collection; K. Skorupska: Manuscript writing, data analysis; J. Młodawski: statistical analysis, data analysis; M. Misiek: data analysis, supervision;W. Rokita': project development;T. Rechberger: drafting and revising the article, supervision.

\section{REFERENCES}

1. Castro DC, Robinson D, Bosch R, et al. Patient-reported outcome assessment. In: Abrams P, Cardozo L, Wagg A, Wien A ed: Incontinence, 6th edn. 2017: 541-598.

2. Tsang S, Royse CF, Terkawi AS. Guidelines for developing, translating, and validating a questionnaire in perioperative and pain medicine. Saudi J Anaesth. 2017; 11(Suppl 1): S80-S89, doi: 10.4103/sja.SJA_203_17, indexed in Pubmed: 28616007.

3. Kelleher CJ, Cardozo LD, Khullar V, et al. A new questionnaire to assess the quality of life of urinary incontinent women. Br J Obstet Gynaecol. 1997; 104(12): 1374-1379, doi: 10.1111/j.1471-0528.1997.tb11006.x, indexed in Pubmed: 9422015.

4. https://iciq.net/iciq-ui-sf.

5. Dybowski B. Badanie urodynamiczne w uroginekologii. In: Barcz E. ed. Uroginekologia. Schorzenia dna miednicy. Via Medica, Gdańsk 2017.

6. King's Health Questionnaire. https://eprovide.mapi-trust.org/instruments/king-s-health-questionnaire.

7. Bump RC. The POP-Q system: two decades of progress and debate. Int Urogynecol J. 2014; 25(4): 441-443, doi: 10.1007/s00192-013-2262-0, indexed in Pubmed: 24504065.

8. Guilford JP. Fundamental Statistics in Psychology and Education. The American Journal of Psychology. 1944; 57(1): 117, doi: 10.2307/1416874.

9. Liang CC, Hsieh WC, Huang L. Outcome of coexistent overactive bladder symptoms in women with urodynamic urinary incontinence following 
anti-incontinence surgery. Int Urogynecol J. 2017; 28(4): 605-611, doi: 10.1007/s00192-016-3153-y, indexed in Pubmed: 27678141.

10. ChappleCR. Primer:questionnaires versus urodynamics in the evaluation of lower urinary tract dysfunction-one, both or none? Nat Clin Pract Urol. 2005; 2(11): 555-564, doi: 10.1038/ncpuro0339, indexed in Pubmed: 16474599.

11. Viana R, Viana S, Neto F, et al. Adaptation and validation of the King's Health Questionnaire in Portuguese women with urinary incontinence. Int Urogynecol J. 2015; 26(7): 1027-1033, doi: 10.1007/s00192-015-26286, indexed in Pubmed: 25653033.

12. Kelleher C, Cardozo L, Toozs-Hobson P. Quality of life and urinary incontinence. Current Opinion in Obstetrics and Gynecology. 1995; 7(5): 404-408, doi: 10.1097/00001703-199510000-00016.

13. Seckiner I, Yesilli C, Mungan NA, et al. Correlations between the ICIQ-SF score and urodynamic findings. Neurourol Urodyn. 2007; 26(4): 492-494, doi: 10.1002/nau.20389, indexed in Pubmed: 17304520.
14. Nunnally J, Bernstein I. Psychometric theory.; 3rd edition. McGraw-Hill Inc, New York 1994.

15. Rust J, Golombok S. Modern psychometrics: The science of psychological assessment. 2nd edition. Routledge, London 1999.

16. Litwin M. How to measure survey reliability and validity. Sage Publications, London 1995.

17. Malik RD, Hess DS, Christie A, et al. Domain comparison between 6 validated questionnaires administered to women with urinary incontinence. Urology. 2019; 132: 75-80, doi: 10.1016/j.urology.2019.07.008, indexed in Pubmed: 31310769.

18. Gotoh $M$, Homma $Y$, Funahashi $Y$, et al. Psychometric validation of the Japanese version of the International Consultation on Incontinence Questionnaire-Short Form. Int J Urol. 2009; 16(3): 303-306, doi: 10.1111/j.1442-2042.2008.02237.x, indexed in Pubmed: 19207608. 